\title{
Écriture pulvérisée e parole en archipel: annotazioni sulla scrittura di René Char
}

\section{Barbara Bottari}

\section{(2) OpenEdition}

\section{Journals}

\section{Edizione digitale}

URL: http://journals.openedition.org/studifrancesi/7805

DOI: $10.4000 /$ studifrancesi.7805

ISSN: 2421-5856

\section{Editore}

Rosenberg \& Sellier

\section{Edizione cartacea}

Data di pubblicazione: 1 juillet 2009

Paginazione: 351-363

ISSN: 0039-2944

\section{Notizia bibliografica digitale}

Barbara Bottari, «Écriture pulvérisée e parole en archipel: annotazioni sulla scrittura di René Char»,

Studi Francesi [Online], 158 (LIII | II) | 2009, online dal 30 novembre 2015, consultato il 09 janvier 2021. URL: http://journals.openedition.org/studifrancesi/7805; DOI: https://doi.org/10.4000/studifrancesi. 7805

\section{(c) (i) (9)}

Studi Francesi è distribuita con Licenza Creative Commons Attribuzione - Non commerciale - Non opere derivate 4.0 Internazionale. 


\section{Écriture pulvérisée e parole en archipel: annotazioni sulla scrittura di René Char ${ }^{1}$}

\section{La scrittura ${ }^{2}$}

En poésie c'est seulement à partir de la communication et de la libre disposition de la totalité des choses entre elles à travers nous que nous nous trouvons engagés et définis, à même d'obtenir notre forme originale et nos propriétés probatoires.

Formule XXI, Partage formel

(1) Questo articolo è il primo di uno studio più ampio composto da tre parti (il primo dall'omonimo titolo, il secondo René Char e 'le raccourci fascinateur' e il terzo Forme tradizionali e innovative nella poesia di René Char) che rendono conto delle caratteristiche più salienti dello stile di René Char ripercorrendo le tappe della scrittura con le sue costanti, delle figure retoriche più utilizzate e delle forme dell'espressione a cui ricorre il poeta per veicolare il suo canto.

Le referenze si riferiscono per la maggior parte all'edizione della «Bibliothèque de la Pléiade» del 1983 (Euvres Complètes, a cura di J. RoudAuT, con la collaborazione per Variantes et Bibliographie di L. e F. A. Jamme, per le Notes e la Description d'un carnet gris di T. Jolas, per la Chronologie, Variantes e Notes di Le Marteau sans mâtre e Dehors la nuit est gouvernée di A. ReInBold, Paris, Gallimard, 1983 («Bibliothèque de La Pléiade», 308, pp. lxxxv-1364), e sono indicate con l'abbreviazione "OC"; per quanto concerne gli scritti dopo questo anno e postumi si fa riferimento all'edizione della «Bibliothèque de la Pléiade» del 1995 (Euvres Complètes, nuova edizione ampliata di testi a cura di J. ROUDAuT, con la collaborazione aggiuntiva di M.-C. CHar e di T. Jolas per la Chronologie e la Bibliographie, Paris, Gallimard, 1995 («Bibliothèque de La Pléiade», 308, pp. lxxxviii-1515), nella quale le opere aggiunte rispettano una numerazione di pagine progressiva rispetto a quella del 1983, e la notazione sarà "OC 1995".

Laddove la raccolta, prima di rientrare in un insieme più ampio, è già stata pubblicata singolarmente, l'anno e l'editore saranno segnalati fra parentesi mentre, a parte, verranno stabilite le date ad quem e post quem della scrittura vera e propria.

Char ha pubblicato buona parte dei suoi scritti da Gallimard ma alcuni anche presso altri editori che saranno riportati con abbreviazioni, fra cui il caro amico Guy Lévis Mano (“G.L.M.”), José Corti ("Corti"), Jean Hugues ("Hugues"), Edwin Engelberts ("Engelberts"), Pierre-André Benoit ("P.
A.B."), Imprimerie Union Paris ("Union”), Éditions Skira a Ginevra ("Skira").

Per le raccolte di aforismi e di frammenti, ho preferito la notazione generica di "formule" seguita dal numero (romano o arabo) corrispondente, senza operare nelle note a piè di pagina alcuna differenziazione, considerandolo termine più ampio che può contenere entrambe le strutture. I Feuillets d'Hypnos costituiscono un caso a parte poiché, oltre a portare la marca di Fogli nel titolo, sono classificati come una forma extragenere dallo stesso autore nella préface e ulteriormente ribattezzati notes.

(2) L'analisi effettuata sulle forme dell'espressione si rivolge specificatamente a quella che Genette definisce «une spatialité en quelque sorte primaire ou élémentaire» (G. GENETTE, La littérature et l'espace in G. Genette, Figures II, Paris, Seuil, 1969, p. 44), ovvero quella del linguaggio che si manifesta attraverso le risorse visuali della grafia, della mise en page, del bianco tipografico, del tipo di carattere utilizzato, dei rapporti orizzontali e verticali che i segni stabiliscono con gli elementi vicini nell'ambito della pagina del testo, «la disposition atemporelle et réversible des signes, des mots, des phrases, du discours dans la simultanéité de ce qu'on nomme un texte» (Ivi, p. 45). A questa indagine ristretta all'iconicità dei poèmes e al parallelismo che si crea con i contenuti, si affianca una breve riflessione anche sulla scrittura nel senso stilistico del termine per osservare da vicino alcune figure retoriche, ovvero intese come «forma che prende lo spazio e forma che si dà il linguaggio» (Ivi, p. 47). Le figure, effet de sens (Ivi, p. 46), restituiscono «l'espace sémantique qui se creuse entre le signifié apparent et le signifié réel abolissant du même coup la linéarité du discours» (Ivi, p. 47) e, in ragione di questa loro potenzialità, intessono rapporti di analogia o di contrasto con le forme del contenuto. Lo stile è preso in considerazione, dunque, come spazialità semantica del discorso letterario: questo stesso è dotato di une épaisseur di senso che nessuna durata può sconfiggere o esaurire. 
L'esistenza di un canone letterario ha suscitato costantemente il tentativo di sovvertirlo e di generare, con questa azione contro le applicazioni servili, una rivolta. Quanto più un poème si libera della tradizione, tanto più si allontana dall'organizzazione coerente degli elementi formali e, in questo distacco, ravviva il linguaggio poetico e le regole che lo disciplinano. Questo fenomeno caratterizzante molta poesia contemporanea segna profondamente i versi di Char, fregiandoli, simultaneamente, di modernità, di originalità e di iconoclastia moderata. Fin da Arsenal (1927-29) opta per una espressione formalmente éclatée in lotta con le forme acquisite. Fra il 1930 e il 1934, anni di militanza surrealista, i poèmes metteranno in atto attraverso il loro aspetto formale questa rivoluzione; seguono i testi programmatici e violentemente rapidi di Moulin premier che, tramite dei processi di élagage e affermissement textuels, offrono le primizie di quelli che saranno i suoi modelli preferiti: l'aforisma, il frammento succinto e il verso condensato. Anche quando la poesia matura e ritorna a strutture più tradizionali, l'estrema compressione del linguaggio interrompe qualsiasi linea di continuità con il passato. Ciascun poème racchiude in sé digressioni e/o ripetizioni che rimangono nell'ambito del poème stesso o, altre volte, travalicano le raccolte e intrattengono una conversazione a più riprese, sempre servendosi del verso lacunaire. I precursori (nonché maestri) di Char si intravedono facilmente se si esamina la forma: Eraclito di Efeso, Lautréamont, Baudelaire, Rimbaud, Apollinaire, Mallarmé, tutti personaggi che rifiutano le formes figées in voga. In particolare, Rimbaud disintegra il testo e ne fa una suite di frammenti isolati nella presentazione visiva e nel significato, ardui da svelare e da render espliciti, sia singolarmente considerati, che nel loro insieme. Negli impulsi del linguaggio e nel movimento dinamico delle frasi interrotte e rapide di Une Saison en enfer, non è possibile rintracciare un contenuto ben definito. Eppure questa accumulazione tourbillonante di righe, dai ritmi precipitati e inaspettati, descrive precisamente l'itinerario dell'artista costellato da successi e da delusioni, da sregolatezza e da lacerazione continue. Il lettore è inglobato in questo spazio poetico, fatto di versi ambigui e desueti per la loro veste tipografica, e così condotto in un viaggio di quête spirituale e immaginario verso l'epilogo di un'esperienza travolgente e radicale. Per una poesia che vuole esprimere questi risultati, il verso e le strutture reçues sono insufficienti.

Char raccoglie la sfida e opta per l'arte concisa, ermetica ma anche, dopo il 1934, eterogenea e costantemente ai margini di qualsiasi scuola letteraria precostituita $^{4}$, operante attraverso l'isolamento del verso poetico, il restringimento radicale dell'espressione, la polverizzazione del poème in piccoli frammenti-entità, lo sbriciolamento dei suoi diversi contenuti accostati in modo insolito per accrescerne la densità, lo stile nominale e conciso, le numerose frasi prive di verbo accumulate e mai concluse (tratti caratteristici dei primi poèmes di Le Marteau sans maître). Char

(3) Partage formel, Seuls demeurent, Fureur et mystère, OC, p. 160.

(4) Si veda La lettre hors commerce ad André Breton del 1947: «Mon cher André, Je te remercie de m'avoir adressé tes projets d'Exposition. [...] Où en suis-je aujourd'hui? Je ne sais au juste. [...] C'est te dire que si certains prodiges ont cessé de compter pour moi, je n'en défends pas moins, de toute mon énergie, le droit de s'affirmer prodigieux. Je ne serai jamais assez loin, assez perdu dans mon indépendance ou son illusion, pour avoir le cœur de ne plus aimer les fortes têtes désoibéissantes qui descendent au fond du cratère, sans se soucier des appels du bord. Ma part la plus active est devenue... l'absence. Je ne suis plus guère présent que par l'amour, l'insoumission, et le grand toit de la mémoire. [...] La transvaluation est accomplie. [...] Je ne peux pas aimer deux fois le même objet. Je suis pour l'hétérogénéité la plus étendue. Que l'homme se débrouille avec les nombres que les dés lui ont consentis. [...] Le vrai secours vient dans le vague. Tu peux faire figurer à cette Exposition "qui je fus" en 1930-1934. [...] Nous avons et saurons toujours nous retrouver côte à côte, à la seconde excessive de l'essentiel. Notre particularité consiste à n'être indésirables qu'en fonction de notre refus de signer le dernier feuillet, celui de l'apaisement. Celui-ci s'arrache - ou nous est enlevé» (I. Pauvreté et privilège, Recherche de la base et du sommet, OC, pp. 660-661). 
si concentra su un verso poetico in cui la potenza della suggestione è maggiore rispetto ai versi tradizionali. Degno discendente dei trovatori provenzali, si adopera a concentrare il suo schema compositivo al massimo, quasi che ridurre lo spazio significhi, in fondo, conferirgli una più grande rilevanza: ciascun verso, ricco di ellissi, contraddizioni, ambiguità, è uno spazio ineludibile e da analizzare a fondo per arrivare a svelare l'enigma. Analogamente a quello che accade a livello delle forme del contenuto, nella poesia chariana l'eloquenza cede il passo alla reticenza, la verbosità al mutismo sviante ma suggestivo. Char conquista questo spazio, paradossalmente, frammentandolo a livello di unità di versi, a livello di significati, a livello di sguardo d'insieme della pagina scritta. Non a caso una delle norme per l'attuazione della scrittura della rivolta prevede la separazione, la distinzione, la messa a distanza, la mutilazione nonché lo smembramento. Forma e contenuto procedono all'unisono in ciascuna delle declinazioni metriche libere o tradizionali (rarissimi questi casi, come Le Deuil des Névons ${ }^{5}$ dove vi è un inizio con rime relativamente regolari e con schema ABAB | CDCD | ECF ma dalla strofa successiva la struttura éclate in GCHHC I IJK e cambia totalmente assetto fino al finale senza accordo di rima). Questo aspetto solido e immutevole della versificazione di René Char, che si costituisce a partire dai blocchi di testo assurti a unità come pure del poème éclair della maturità, mostra la pazienza del poeta in quanto artefice e artigiano dei valeurs poétiques tenables ${ }^{6}$, della pyramide du Chant che va costruendo en terrasses successi$v^{7} s^{7}$. Questo concetto ben identifica uno stratificarsi di spazi conquistati progressivamente e per gradi. Lo scrittore lo riprende ben tre volte nella sua opera: la prima volta negli anni'30 assumendo il qualificativo di poète terrassier ${ }^{8}$, successivamente come regola programmatica in Moulin premier (come recita la precedente citazione) e, infine, riconfermandolo come una condizione imperitura dei suoi poèmes in una breve introduzione al collage poetico di Le Batôn de rosier degli anni '80 (Ces poèmes ne sont pas retenus ici par choix. Longtemps ils restèrent méditatifs, mais «en terrasses» ${ }^{9}$ ).

La stessa abilità Char l'apprezzava nell'alleato Georges Braque: da pittore cubista insisteva a voler penetrare nello spazio più profondo e recondito dei suoi soggetti per giungere, senza mai tradirli, «alla loro notte», «alla loro nudità primigenia» ${ }^{10}$, alla loro trasparenza nascosta. Se l'artista interveniva, manipolando la realtà, era unicamente per far emergere meglio la bellezza latente insita in ogni oggetto, per svelarne l'estrema diversità e «la perspicacia» ${ }^{11}$. I frammenti di una tela di pittura cubista, offrendo l'immagine di una entità vista simultaneamente da diverse angolazioni, consentono di cogliere, per un processo di deformazione-ricostruzione, il fascino intrinseco della totalità così scandita. Le parole sono manipolate con discrezione nel significante e nel significato; per quanto riguarda il significante, l'uso del corsivo o delle maiuscole invita a enfatizzare l'attenzione verso il termine:

Qu'à toute réquisition un poème puisse efficacement en tout comme en fragments, parcours entier, se confirmer, c'est-à-dire assortir ses errements, m'apporte la preuve de son indicible réalité. Décampe, interdit de ses fondations... Aime, riveraine. ${ }^{12}$

(5) La bibliothèque est en feu et autres poèmes, $\mathrm{La}$ Parole en archipel, OC, pp. 389-391.

(6) Formule XII, Partage formel, Partage formel, Seuls demeurent, Fureur et mystère, OC, p. 158

(7) Ibidem.

(8) Al plurale in Les soleils chanteurs, L'Action de la justice est éteinte, Le Marteau sans maître suivi de Moulin premier, OC, p. 27.
(9) Le Bâton de rosier, OC, p. 787.

(10) 2. Sous la verrière, En vue de Georges Braque, II. Alliés substantiels, Recherche de la base et du sommet, OC, p. 675.

(11) Ibidem.

(12) Formule LXV, Moulin premier, Le Marteau sans mâtre suivi de Moulin premier, OC, p. 78. 
Enfants d'Espagne - ROUGES, oh combien, à embuer pour toujours l'éclat de l'acier qui vous déchiquette; - À Vous. [...] Enfants d'Espagne, [...] Enfants d'Espagne, j'ai formé de PLACARD alors que les yeux matinals de certains d'entre vous $[\ldots]^{13}$

Per quanto concerne il significato, Char vuole ottenere lo stesso effetto con forme nuove e scomposte, con un linguaggio che si nutre di termini desueti e inusitati perché classificati come "vieux" (souchetée ${ }^{14}$, riesumato dalla metà del XIX secolo, che riporta in sé il termine "la souche”, ossia il ceppo dell'albero, simbolo della radice, del radicamento alle origini e della provenienza, tema ricorrente e significativo) o "vieillis" (la locuzione à la réserve de $e^{15}$ ) o parole che designano una cosa del passato, scomparsa (faneur). Il poeta si concentra su temi specialistici con vocaboli prettamente appartenenti al mondo vegetale (les sarments, l'ipomée, la primevère, les ergots, la centaurée, la renouée, l'armoise, le varech) o a quello animale (le frelon, la pariade) poco frequenti in poesia, o a quelli legati al mondo rurale, dei contadini (aoûtement, la fenaison, le billot, l'aven) e della semina (le limon, la pioche, la chaume), o all'ambito della navigazione (le rostre, béler, bourlinguer) e della pesca (le harpon). In altre occasioni, esporta parole da campi insoliti (balandran - balandron - balandrane dalla sfera agreste) o esibisce termini di personalissimo conio (come la parola vomito, dall'intuibile valenza negativa; altro neologismo è pisse-lyres ${ }^{16}$ sul modello pisse-copie, «écrivain abondant et médiocre»). In definitiva, il linguaggio evita le banali reiterazioni del senso abituale dei lemmi e della ripetizione di luoghi comuni. Il desiderio è di riformulare e di ridire con un Verbo dalle fisionomie mutevoli e dai poteri moltiplicatori di suggestioni, un Verbo che abita un "poème-dedalo" dalle destinazioni impreviste e imprevedibili.

\section{Le costanti}

Le poète fait éclater les liens de ce qu'il touche. Il n'enseigne pas la fin des liens.

À faulx contente ${ }^{17}$

La scrittura poetica di René Char è caratterizzata da brevità e da velocità: il ritmo estremamente rapido, sulla scorta di André Breton che, ne L'Amour fou, insisteva: «La beauté sera convulsive ou ne sera pas ${ }^{18}$. Char segue anche un altro maestro, Arthur Rimbaud, che si distingue per la "dialettica ultrarapida" ${ }^{19}$. Il poeta deve affrontare il mondo con urgenza impellente (Tu es pressé d'écrire $e^{20}$ ), dato che les mondes éloquents ont été perdus e i frammenti di bellezza sopravvissuti devono esser mantenuti. La rapidità si scorge nell'immagine, in maniera analoga a come era trattata dai Surrealisti ${ }^{21}$, ma anche nella forma del frammento, dell'aforisma o di versi brevi martelés, come:

(13) Dédicace, op. cit., OC, p. 89.

(14) Le bulletin des Baux, Le Poème pulvérisé, Fureur et mystère, OC, p. 258.

(15) VIII. Aux miens, Neuf merci pour Vieira da Silva, La bibliothèque est en feu et autres poèmes, $L a$ Parole en archipel, OC, p. 387.

(16) Tu as bien fait de partir, Artbur Rimbaud, La Fontaine narrative, Fureur et mystère, OC, p. 275.

(17) OC, p. 783.

(18) Paris, Gallimard, 1937, p. 21.

(19) E ancora: «[...] sa découverte, sa date incendiaire, c'est la rapidité. [...]» (Arthur Rimbaud in III. Grands astreignants ou la conversation souveraine, Recherche de la base et du sommet, OC).

(20) II, Commune présence, Moulin premier, Le Marteau sans maître, OC, p. 80 .

(21) «L'image surréaliste n'est pas un produit extraordinaire: c'est la vitesse maximum qu'on ait atteinte, et semble-t-il qu'on puisse atteindre, dans la transmission des images par la langue» (G. MouNIN, La communication poétique précedé de Avezvous lu René Char?, Paris, Gallimard, 1969, «Les Essais», p. 96). 
Visage, chaleur blanche,

Sœur passante, sœur disant,

Suave persévérance,

Visage, chaleur blanche ${ }^{22}$.

Si tratta di una poesia ellittica, che usa strumenti mirati e punta sul rigore dei suoi meccanismi. L'economia dei suoi legami logici provoca dei corto-circuiti nella poesia, da cui emerge l'éclair poetico ${ }^{23}$. Al discorso o al commento, l'artista ama alternare il raccourci fascinateur. Lo dimostrano anche i titoli che oscillano fra la sintesi più estrema e misteriosa, come Éprise $e^{24}$ o Sociéte $e^{25}$, e la verbosità più curiosa e singolare poiché difficilmente ricollegabili ${ }^{26}$ con il resto dei titoli o il contenuto dei poèmes e della prosa poetica (per esempio, Comment te trouves-tu là? Petite marmite, mais tu es blessée! $!^{27}$ titolo di una raccolta dove, fra i tanti argomenti trattati, è celebrata l'unione dell'uomo con la natura e il disprezzo per il Maître Mécanicien ${ }^{28}$ ).

Le forme dell'espressione sono ben definite nella poesia chariana e in stretta connessione dialettica fra loro. Char è il poeta dell'aforisma, del noeud o dell'éclair, ma anche il poeta di una retorica esplicitata, di una forza d'espansione, di cui il poème en prose è portatore. Se l'aforisma e il frammento sono il primo polo dialettico, l'altro è costituito dal poème en prose, dal poème en vers e dalla prosa poetica (o poetizzata). Nella Mise en garde de La Sieste blanche ${ }^{29}$, un nous locuteur invita a identificare, a classificare i testi poetici in base alla loro collocazione: «Nous avons sur notre versant tempéré une suite de chansons qui nous flanquent, [...]». Questo pronome lega strettamente la versificazione al cammino e gli riconosce, come principale virtù, la transitività fra gli stati o fra i regni (appannaggio, un tempo, del dio Ermes). Ciascun "a capo" è un nuovo impulso, che porta ad avanzare, apre verso un'immagine inattesa, stabilisce nuove connessioni e svia il lettore da qualsiasi poetare tradizionale ${ }^{30}$. A volte, il poème in versi si restringe fino a diventar un haikai, proprio perché poche parole si rivelano sufficienti per far emergere l'emozione poetica dalla realtà quotidiana; assomiglia all'arte dell'istantanea, che coglie l'eterno nel cuore dell'effimero, dipingendo il reale sfuggente, difficilmente fissabile e generato dallo sguardo sul mondo. Il poeta parla della vita semplice, à fleur de terre, dando più voce al nascosto, al minimo, a tutto ciò che sait tirer parti de l'éternité d'une olive. L'artista unisce i frammenti sparsi di realtà e dà così forma alla continuità ${ }^{31}$. Dal frammento, prolungatosi e ampliatosi, si scivola verso la forma del poème in versi, che può evolvere in più pagine (come succede nel caso di Le Visage nuptial ${ }^{32}$ ), senza disperdere né forza né densità. Da quest'espansione, il passo verso il

(22) Sur le volet d'une fenêtre, Les Loyaux Adversaires, Fureur et mystère, OC, p. 239.

(23) In un'intervista con F. HuSER, René Char sottolineava: «Je ne brûle pas les relais, mais je les élude. De cette spoliation est né le poème bref» (Sous ma casquette amarante, OC, p. 823).

(24) Effilage du sac de jute, Fenêtre dormantes et porte sur le toit, OC, p. 621.

(25) Te devinant éveillé pour si peu..., Les Voisinages de Van Gogh, OC 1995, p. 832.

(26) Interessante il contributo di C. Dupouy, René Char, Paris, Les Dossiers Belfond, 1987, 336 pp., nel primo capitolo dal titolo Les titres - faut-il s'y fier?, pp. 153-176 (Qu'est-ce qu'un titre?; Les titres des recueils; Les titres des poèmes; Le cas particulier des poèmes aphoristiques; Autonomie des titres, et organisation des recueils).

(27) Fenêtres dormantes et porte sur le toit, OC, p. 599.
(28) Comment ai-je pu prendre un tel retard?, III. Comment te trouves-tu là? Petite marmite, mais tu es blessée!, op. cit., p. 603.

(29) Les Matinaux, OC, p. 291.

(30) A questo proposito, nell'articolo Victor Brauner, Char trova l'occasione per riaffermare: «Le poète qui versifie en marchant bouscule de son talon frangé d'écume des centaines de mots à ce coup inutiles; de même un vaste ouvrage qui surgit en se construisant alerte et fait pleuvoir d'insolites projectiles. Tous deux taillent leur énigme à l'éclair d'y toucher. En cet air, l'espace s'illumine et le sol s'obscurcit» (II, Alliés substantiels, Recherche de la base et du sommet, OC, p. 683).

(31) P. NÉE, Le sens de la continuité dans l'œuvre de René Char, Thèse de doctorat (nouveau régime), Université de Paris-VII, 1986.

(32) Seuls demeurent, Fureur et mystère, OC, pp. 151-153. 
poème en prose è breve: là, c'è pari complessità e si sviluppa una fitta rete di rapporti, impensati e inediti, che costituiscono la forza e l'innovazione chariane. Ogni composizione rimanda, perciò, a nuovi spazi, spostando in avanti, continuamente, $i$ riferimenti di lettura e rendendo difficile l'interpretazione.

Anche nei poèmes en prose emerge il dinamismo, soprattutto quando la linearità del discorso è interrotta dall'uso frequente di ellissi, di apposizioni e di asindeti. Rileggendo Violences ${ }^{33}$, il discorso narrativo del primo paragrafo («Des pêcheurs d'anguilles venaient là fouiller de leur fer les rares herbes dans l'espoir d'en extraire de quoi amorcer leurs lignes. Toute la pègre des écumes se mettait à l'abri du besoin dans ce lieu. Et chaque nuit le même manège se répétait dont j'étais le témoin sans nom et la victime») è seguito e liquidato da una scrittura paratattica, in un fiorire di frasi brevi («J'optais pour l'obscurité et la réclusion. Étoile du destiné. J'entrouvre la porte du jardin des morts. Des fleurs serviles se recueillent. Compagnes de l'homme. Oreilles du Créateur»). Si vengono a costituire des îlots ${ }^{34}$ di senso o, più precisamente, delle îles de sens, come faceva notare Maurice Blanchot, «[qui] sont plutôt que coordonnées, posées les unes à côté des autres» ${ }^{35}$, che danno vita alla poesia in arcipelago. Questi segmenti, o isole, a volte frammenti densi, a volte versi unitari, sembrano nati da una forza eruttiva, che irrompe senza preavviso dall'oscurità prenatale ${ }^{36}$.

Tutto l'universo di René Char è dotato di immagini celeri, che uniscono gli elementi più disparati, anche "senza rapporto", e fa perno tanto sulla dinamica centrifuga ed esplosiva dell'éclatement nelle diverse forme poetiche scelte, quanto sulla modalità inversa, ossia sulla forza che comprime, che forma il blocco adamantino e infrangibile $e^{37}$ della poesia chariana. L'effetto di éclatement è ancora più accentuato dalla forza, che si manifesta nelle unità semantiche maggiori e nei singoli sintagmi, di natura contraria, centripeta. Come ben chiarisce Agosti, «a una fenomenologia dell'éclatement sul piano della manifestazione lessicale di superficie (il poème pulvérisé) [,] corrisponde o fa contraltare, la fenomenologia - non manifesta ma effettiva e, di fatto, percepita - della coesione del poème (e delle altre forme, n.d.r.) a livello profondo ${ }^{38}$. La composizione poetica si articola, di conseguenza, in base a tre livelli generativi ${ }^{39}$ correlati, partendo dal livello lessicale di superficie, ove si manifesta una tensione esplosiva e centrifuga, che origina le isole del poème pulvérisé, si procede verso un livello più profondo, composto da un reticolo di relazioni semantiche (o semiche), poste in essere dalle entità presenti nella rappresentazione di superficie e dal loro connubio, per terminare, con un terzo livello, identificabile come «un punto cieco del testo». Qui, i nodi del reticolo (individuabili in sistemi d'opposizione) del livello precedente svaniscono e si compongono in figure di senso compatte, ma difficilmente accessibili per il concetto. Queste «figure di senso compatte ma irricevibili per il concetto $\gg^{40}$ sono veicolate, di norma, da elementi del testo ma rappresentano anche il massimo della profondità e il punto più basso è, di regola, scarsamente visibile nel testo.

In Char, dunque, si compie un'operazione insolita, che lo differenzia da tutte le esperienze di tanta parte della poesia contemporanea, come quella di Mallarmé, quel-

(33) Seuls demeurent, Fureur et mystère, OC, p. 130.

(34) G. BuIN, L'instant multiple in «L'Arc», numéro spécial René Char, n²2, 1963, pp. 19-20.

(35) M. Blanchot, L'entretien infini, Paris, Gallimard, 1969, p. 463.

(36) «Pourquoi poème pulvérisé? Parce qu'au terme de son voyage vers le Pays, après l'obscurité prénatale et la dureté terrestre, la finitude du poème est lumière, apport de l'être à la vie» (La biblio- thèque est en feu, La bibliothèque est en feu et autres poèmes, La Parole en archipel, OC, p. 378).

(37) S. Agosti, Figure profonde e figure di superficie nella poesia di René Char in R. CHAR, Canti della Balandrane, Sfilacciatura del sacco di iuta, a cura di Stefano Agosti, Milano, Mondadori, 1993, xx, p. $x$.

(38) Ibidem.

(39) Ivi, p. XxIx

(40) Ivi, p. Xx. 
la della linea petrarchesca della poesia europea, quella di autori come Yeats, Dylan Thomas, Jiménez e García Lorca, ma lo assimila a certe esperienze-limite del primo e del secondo Ottocento, in particolare dell'ultimo Hölderlin e del Rimbaud delle Illuminations e dei Derniers vers. Char si avvicina a questi artisti nella misura in cui tutti e tre condividono la ricerca di un senso, come prodotto di isotopie non grammaticalizzate, ovvero non acquisite all'ordine del discorso, un senso che porti in sé configurazioni concettuali "altre", non in linea con le figure canoniche del pensiero. Anche in questo ambito, Char sembra aver continuato e portato en avant il progetto dei primi due. Per molti poèmes, l'esito è una "dinamica doppia e a orientamento invertito" ${ }^{41}$ che produce una serie di figure di senso, lontane dalla logica normativa. La profondità ultima del testo si rileva sulla sua superficie e, a sua volta, le rappresentazioni di superficie si raccolgono sul fondo indifferenziato della neutralizzazione delle opposizioni e della coabitazione dei contrarât ${ }^{42}$.

La scrittura poetica di René Char inventa uno spazio costruito: avendo il paesaggio come sfondo, i frammenti, i versi, le proposizioni si dispongono en terrasses successives, in modo da fondare un espace de souveraineté, ove "abitano" luoghi, azioni ed emozioni. Questo meccanismo di identificazione è possibile poiché esiste un'equivalenza sicura e comprovata fra il poeta e il poème («Ce qui me console, lorsque je serai mort, c'est que je serai là - disloqué, hideux - pour me voir poème» ${ }^{43}$ ) o fra l'artista e i suoi versi («Sens le mot être à son tour ce que tu es. Et son existence devient doublement la tienne» ${ }^{44}$. L'assimilazione e coincidenza fra creatore e opera esalta la forma dell'espressione ed entrambi gioiscono di proprietà comuni e transitive: la parola chariana ripercuote in sé, nella disposizione sulla pagina bianca, negli a capo e nel tipo di caratteri (in tondo, occasionalmente in corsivo o maiuscoletto), le prerogative del poeta e diventa alternativamente muta, enigmatica, discorsiva, allusiva nonché autentica e piena, profonda di significati. Analizzare l'aspetto formale conduce inevitabilmente alla sostanza interna in una relazione biunivoca che acquisisce un valore sempre crescente via via che si procede nella lettura dell'opera. Ed è proprio in virtù dell'importanza che Char conferisce a questo spazio formale, unitamente alla rilevanza che ha dato a quello contenutistico, che cesella e plasma diverse possibilità combinatorie dei versi: dal poème en vers a quello en prose (tradizionale, rarefatto o en abîme, ossia contenente un ulteriore componimento a sé, come Dédicace $\left.{ }^{45}\right)$, dall'aforisma al frammento, dalla prosa poetica ai più radi esperimenti di récit onirico (Eaux-mères ${ }^{46}$ o Réserve romancée $e^{47}$ ), di annotazione lessicale formato dizionario, alias poème index $x^{48}$ (Sur le franc-bord ${ }^{49}$ concernente il lemma Iris e Le dos tourné, la Balandrane... ${ }^{50}$ per il vocabolo Balandrane) e il poème-théâtre, ovvero un poème in versi liberi simile a una partitura teatrale di cui Fête des arbres et du chasseur è l'esempio più evidente.

(41) Ibidem.

(42) Ibidem.

(43) Les compagnons dans le jardin, La bibliothèque est en feu et autres poèmes, La Parole en archipel, OC, p. 383 .

(44) La scie rêveuse, Dans la pluie giboyeuse, Le Nu perdu, OC, p. 454.

(45) Si tratta di un gioco di mises en abimes che coinvolge anche generi diversi: Lettera amorosa è la prima raccolta di La Parole en archipel ed è una trasposizione in versi dell'omonima composizione musicale di Claudio Monteverdi a sua volta tratta dal balletto Tirsi e Clori. Lettera amorosa inizia con Dédicace, una lunga prosa poetica in cui spicca il poème en vers Chant d'Insomnie (pp. 339-346).

(46) Abondance viendra, Le Marteau sans maître suivi de Moulin premier, OC, pp. 50-53.

(47) Formule XXVII, Moulin premier, op. cit., pp. 68-69.

(48) Si rimanda alla trattazione approfondita che fa R. LANCASTER di questa forma singolare nel capitolo "Solitaire et multiple": l'éclatement du mot della sua monografia La poésie éclatée de René Char, Amsterdam-Atlanta, GA, Rodopi, 1994 («Faux Titre»; $80)$, pp. 28-40.

(49) Lettera amorosa, La Parole en archipel, OC, pp. 346-347.

(50) Le train martyr, Chants de la Balandrane, $O C$, pp. 571-572.

(51) Les Matinaux, OC, pp. 280-288. Ci sono altri casi di poème théâtre: Sosie (Arsenal, Le Marteau sans maitre suivi de Moulin premier, OC, p. 13), Les Trans- 
Éclatement de sens, épanouissement, dissémination sono le attività essenziali di queste forme dell'espressione che si propagano in diverse direzioni, riuniscono in sé i temi più contraddittori, danno vita al connubio di immagini dissimili, propongono inedite combinazioni linguistiche e generano una varietà di senso e di toni considerevole. La parola, una volta rievocata, si moltiplica di significazioni, si sviluppa, si apre all'infinito, fa andare in mille pezzi i limiti testuali imposti (come ben mostrano, in particolare, i poèmes index) e si esibisce in costellazioni, in arcipelaghi, in isole, in ammassi di versi. Il poeta soddisfa il suo bisogno di conoscenza illimitata conquistando da révolté anche lo spazio grafico, più tangibile e concreto, della pagina.

\section{Metonimia, sineddoche e metafora}

Charbonnier nous offre l'image de sa route intérieure. À peine parcourue, sa générosité la rompt et nous la rend comme les deux bras d'un pain délibéré.

Pierre Charbonnier ${ }^{52}$

L'uso sistematico di questi tre tropi nella poesia di Char è sintomatico del tipo di percorso intrapreso dall'autore. Queste tre figure agiscono sulla categoria dell'immagine in modo simile ma, essendo diversa la natura del rapporto esistente fra comparante e comparato, sostanzialmente sono animate da scopi finali diversi. Sfruttando queste differenze, lo scrittore vuole mostrare le tre fasi salienti della sua poesia a livello formale, con ampie conseguenze sul livello contenutistico: innanzittutto, la fissuration, messa in atto dalla metonimia, è seguita dall'essaimage perpetrato, in modo più incisivo, dalla sineddoche per finire con la metafora che, nei suoi svariati modi, ricompone in un tissage quello che era stato separato e scisso. Il susseguirsi di questi tre passaggi segna tutta la scrittura di Char e permette di tracciare un itinerario in questa produzione complessa.

La metonimia, figura di trasferimento semantico fondata sulla relazione di contiguità logica e/o materiale fra il termine letterale e il termine traslato, lega un elemento a un altro simile per una relazione di causa/effetto, materia/oggetto, contenente/contenuto, astratto/concreto e concreto/astratto, mezzo/persona, autore/opera, ma si tratta sempre di due termini appartenenti allo stesso insieme, che coincidono, in un certo senso, e che si trovano a essere messi in relazione per causa/effetto e per tutti gli altri binomi appena citati. Si rimane dunque sullo stesso asse sintagmatico e l'affinità può essere spaziale, causale o temporale. Char, di queste tre relazioni, di solito preferisce la contiguità spaziale fra due elementi: in Biens égaux $x^{53}$, inserisce une route de lavande et de vin ma il paesaggio che aveva di fronte agli occhi, verosimilmente, è un chemin costeggiato dalle piante di lavanda e dalle vigne (con uso doppio della metonimia, ovvero anche del prodotto per la pianta). L'immagine prodotta è sotto il segno dello scambio, il che consente una sorta di passaggio, di dinamizzazione e di riunione di due elementi spesso diversi che diventano complementari. Nella maggior parte dei casi per Char le unità contigue sono in relazione di opposizione, in virtù della struttura eraclitea del suo universo. Vive, dunque, questa figura come una unificatrice nuzia-

parents (La Sieste blanche, Les Matinaux, OC, pp. 295 302), Prévaricateur (Newton cassa la mise en scène, Chants de la Balandrane, OC, p. 546), Le crépusule est vent du large (op. cit., pp. 546-547), Le condamné (Les Voisinages de Van Gogh, OC 1995, p. 833) e Cabane
(Éloge d'une Soupçonnée, OC 1995, p. 844).

(52) II. Alliés substantiels, Recherche de la base et du sommet, OC, p. 684.

(53) Biens égaux, Le Poème pulvérisé, Fureur et mystère, OC, pp. 251-252. 
le che permette l'inversione dei contrari. Sempre nel citato poème, il ribaltamento dei ruoli maschile e femminile, preparato dalla metonimia, è ben definito: l'uomo diventa passivo ed è il Tu femminile che agisce. La strada è intrapresa, il fragile equilibrio in cui i due poli erano uguali vacilla. Dopo la prossimità della Rencontre, che dura solo un éclair (l'angle fusant), la coppia avanza e si ritrova inversamente disposta. Lavanda, l'amante, è passata dalla parte del vino (vir), che, a sua volta, ha preso il posto della prima: «[...] Sur une route de lavande et de vin, nous avons marché côte à côte dans un cadre enfantin de poussière à gosier de ronces, l'un se sachant aimé de l'autre. Ce n'est pas un homme à tête de fable que plus tard tu baisais derrière les brumes de ton lit constant $[\ldots] \gg^{54}$. C'è inversione: l'uomo ha acquisito la tête di donna. Angoscia e fiducia, defezione e coraggio, rivaleggiano senza annullarsi, avendo ognuna, a turno, la meglio, senza una vittoria definitiva. La polvere si appropria della gola che la respira e la sofferenza del respiro è espressa dalla sua causa figurata, les ronces. In un altro contesto, la metonimia mostra la felicità dell'armonia in cui i contrari si scambiano qualità nell'allegria come nel poème Allégresse:

Les nuages sont dans les rivières, les torrents parcourent le ciel. Sans saisie les journées montent en graine, meurent en herbe. Le temps de la famine et celui de la moisson, l'un sous l'autre dans l'air haillonneux, ont effacé leur différence. Ils filent ensemble, ils bivaquent! comment la peur serait-elle distincte de l'espoir, passant ravinéess

Si tratta dello stesso universo di fecondità di Jacquemard e Julia in cui la persistenza della famine e della paura consente di meglio apprezzare rispettivamente la moisson e la speranza. I contrari comunicano senza abolirsi in un dualismo essenziale, nuziale che è promessa di prosperità. Malgrado ciò, l'accordo metonimico è fragile e rischia in ogni momento di mostrare la sua déchirure attraverso il poème. L'alleanza felice è rara, frutto di nozze effimere, è la faille che trionfa, concretizzata nella scrittura poetica che è per sua natura breve saetta che invade lo spazio bianco.

La sineddoche, se frattura si è prodotta, non fa altro che accentuarla poiché intacca il tutto frammentandolo in una moltitudine di piccole parti. Come la metonimia, è figura di contiguità ma in una relazione di maggiore o di minore estensione in cui si intende rappresentare la parte per il tutto o viceversa, la parola di significato più ampio per quella più ristretta, il genere per la specie e viceversa, il singolare per il plurale e viceversa, ovvero in rapporti particolarizzanti o generalizzanti. Char predilige la sineddoche che è particolareggiante e sfrutta a fondo lo smembramento del corpo, maschile o femminile, più spesso dell'altro personaggio presente nel poème poiché il Je si mantiene più discreto, Fugitif harmonieux, à la personne à peine indi$q_{u e ́} e^{56}$. Come in un quadro cubista che osserva una particolarità da diversi punti di vista, questo tropo intacca la schiena, le spalle (punti deboli dello stesso autore) o il sesso, la bocca, gli occhi, il viso, le braccia, il polso, a seconda che si tratti di uomini o di donne. Il maschio visto di schiena compare alla fine del periodo surrealista in cui questa poesia ritorna a una visione più concreta e diventerà sempre più una costante, anche in ragione del dato biografico. È l'immagine di una persona che si allontana, il Rimbaud archetipo del poeta che se ne va tournant un dos maçonné aux activités

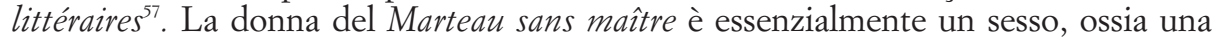
ferita, emblematica della metonimia, legame e rottura, poiché indica sia la prossimità

(54) Ivi, p. 251.

(55) L'allégresse, Quitter, La Parole en archipel, $O C$, p. 415.

(56) L'éclairage du pénitencier, Seuls demeurent,
Fureur et mystère, OC, p. 144.

(57) Arthur Rimbaud, III. Grands astreignants ou la conversation souveraine, Recherche de la base et du sommet, OC, p. 728. 
che la separazione e la possibilità di scambio. Questa concezione del corpo femminile ha in sé una dimensione sadica, come testimonia il poème dal titolo Sade, l'amour enfin sauvé de la boue du ciel, cet héritage suffira aux hommes contre la famine:

Le pur sang ravi à la roseraie

Frôleuse mentale en flambeau

Si juteuse le crin flatté

L'odorat surmené à proximité d'une colonie de délices ${ }^{58}$.

In seguito, avviene uno spostamento di interesse e di organo e la rottura avviene all'altezza del polso: separate dalla scrittura di quindici anni, Eva d'Autun dal poignet sectionné e la giovane donna mutilata di L'Inoffensif si fanno eco. La mano, simbolo di un possibile legame, è tranciata e resta solo il polso, a volte isolato, un moncone che raffigura la morte per le sue due estremità troncate. Non è un mutilare vano ma funzionale all'espressione di qualcosa che sta molto a cuore a Char: la sineddoche è quasi metafora di scrittura nel comunicare che nel poème rimangono solamente delle tracce della straordinaria Rencontre con la poesia poiché l'evento, come l'éclair, è polverizzatore di entrambi i convitati. La violenza dello choc è proporzionale alla sua intensità. Lo raffigura con più esattezza il poème Congé au vent in cui la fanciulla, simbolica in ogni senso, è ridotta alle braccia, alla schiena e alle labbra (vi sono anche altre parti umane appartenenti all'ipotetico lettore):

À flancs de coteau du village bivouaquent des champs fournis de mimosas. À l'époque de la cueillette, il arrive que, loin de leur endroit, on fasse la rencontre extrêmement odorante d'une fille dont les bras se sont occupés durant la journée aux fragiles branches. Pareille à une lampe dont l'auréole de clarté serait de parfum, elle s'en va, le dos tourné au soleil couchant.

Il serait sacrilège de lui adresser la parole.

L'espadrille foulant l'herbe, cédez-lui le pas du chemin. Peut-être aurez-vous la chance de distinguer sur ses lèvres la chimère de l'humidité de la Nuit? ${ }^{59}$

Il poème non può che rappresentare alcuni frammenti di poesia e mai la totalità che si configura come sfuggente e impossibile, illusoria se fosse descritta in tutte le sue parti. Si svelerebbe quel mistero che deve permanere perché la poesia continui a esistere. Il movimento di fuga è doppio poiché il poeta è colui che "prende congedo" e si allontana seguendo le tracce di colei che gli sfugge da sempre ma sa bene che per trovarla, novello Orfeo, le deve voltare le spalle, rischiando di perderla di vista. Forse Char suggerisce all'artista che solamente annullandosi può far avanzare la poesia, nel vuoto del suo corpo evanescente e spezzettato, così come lo rappresenta in Le Bois de l'Epte:

Je n'étais ce jour-là plus avant que deux jambes qui marchent.

Aussi, le regard sec, le nul au centre du visage,

Je me mis à suivre le ruisseau du vallon.

Bas coureur, ce fade ermite ne s'immisçait pas

Dans l'informe où je m'étendais toujours plus avant.

Venus du mur d'angle d'une ruine laissée jadis par l'incendie,

Plongèrent soudain dans l'eau grise

(58) Poèmes militants, Le Marteau sans mâttre suivi de Moulin premier, OC, p. 40.

(59) Congé au vent, Seuls demeurent, Fureur et mystère, OC, p. 130. I corsivi di questa citazione e delle successive sono nostri. 
Deux rosiers sauvages pleins d'une douce et inflexible volonté.

Il s'y devinait comme un commerce d'êtres disparus, à la veille de s'annoncer encore.

Le rauque incarnat d'une rose, en frappant l'eau,

Rétablit la face première du ciel avec l'ivresse des questions,

Éveilla au milieu des paroles amoureuses la terre,

Me poussa dans l'avenir comme un outil affamé et fiévreux.

Le bois de l'Epte commençait un tournant plus loin.

Mais je n'eus pas à le traverser, le cher grainetier du relèvement!

Je humai, sur le talon du demi-tour, le remugle des prairies où fondait une bête,

J'entendis glisser la peureuse couleuvre;

De chacun - ne me traitez pas durement - j'accomplissais, je le sus, les souhaits ${ }^{60}$.

Deux jambes, le regard sec e il centre du visage appartengono al je locuteur completamente assorbito unicamente dalla sua attività che lo reifica (comme un outil affamé et fiévreux) e lo spinge sempre più avanti nello spazio e nel tempo. Anche il paesaggio subisce lo sguardo sineddotico dell'artista: mur d'angle, une ruine, le rauque incarnat d'une rose, un tournant e le talon du demi-tour sono i dettagli che emergono come protagonisti essenziali della vicenda del poeta che ha la missione e la volontà di esaudire la quête (De chacun [...] j’accomplissais, je le sus, les soubaits). La sineddoche non è per Char una semplice figura di stile bensì un principio fondatore della sua scrittura. Privilegiando la nozione di sfrondamento, diremmo di essaimage, l'autore concepisce il suo lavoro come un'attività che consiste nell'eliminazione di tutti quegli elementi superflui conservando unicamente i punti nodali ed essenziali (come mostrano le diverse parti del corpo), anch'essi ridotti al minimo. Ecco uno dei motivi della brevità e del raccourci fascinateur che si staglia sulla pagina bianca da dominare. Queste due figure, infatti, danno materialità a quella scrittura della rivolta che prevede il dominio dello spazio e non mancano di separare solo per meglio controllare forme (e, di riflesso, contenuti): sono inserite in un programma ben preciso di regole. Difatti, procedendo per allusione, non facendo mai menzione della totalità, la sua scrittura si basa su una economia di tipo sineddotico per restringere in uno spazio ben delimitato il più grande numero di dati possibili e per moltiplicare le referenze ellittiche (al mondo reale, alla mitologia antica, alla filosofia presocratica ed esoteristaalchemica) dotate di una precisione meticolosa. Questa concentrazione spaziale nelle forme dell'espressione è sicuramente in aperto contrasto con l'ampliamento dello stesso a livello delle forme del contenuto ma tutta la poesia di Char vive di (o convive con) queste contraddizioni, palesi o nascoste. Lo scopo è riuscire ad aprire attraverso il poème su tutto un mondo reale e culturale. La sineddoche è, quindi, legame ma anche rottura: scomporre significa anche fare proprio, mettere in evidenza e introdurre un dinamismo in ciò che era una realtà assopita e diffusa. Più è netta e violenta, più è riuscito l'atto poetico comunicativo: il poeta si deve distinguere dal mondo che lo circonda per poterlo modificare radicalmente, per ristabilire l'ordine perduto. In questo la poesia è énergie disloquante ${ }^{61}$ che fa a pezzi la parola: il poème si compone di pieno (ciò che si presenta come testo) e di vuoto (ciò che sembra la negazione stessa della parola) ed è proprio quel vuoto a esser più significativo. I vocaboli sono impotenti, banalmente umani, a volte troppo precisi (appartenenti, addirittura, ai microlinguaggi) e talvolta troppo vaghi, ed è proprio quando si sbriciolano che vengono potentemente fecondati, tutti contornati dal bianco tipografico che li minaccia e li corrode fin

(60) Le bois de l'Epte, Poèmes des deux années, La Parole en archipel, OC, p. 371.
(61) Pour un Prométhée saxifrage, Au-dessus du vent, La Parole en archipel, OC, p. 399. 
a farli sparire. Forse questa démarche di rimessa in discussione del linguaggio stesso è sembrata la più logica a un artista che voleva esprimere l'indicibile: ciò che è lontano e innominabile si offre attraverso la fissuration e l'essaimage, frutto del poiein dello scrittore. Gli spazi bianchi lasciati non dimostrano solo una mancanza, un'impotenza a esprimersi ma anche il segno stesso dello scontro con un argomento, la poesia e l'arte, più in generale, nel suo assoluto, che si nasconde da sempre a ogni creatore.

Una volta passato attraverso la metonimia, che fende la parola, e la sineddoche, che la polverizza in mille débris mortels, l'artigiano dei versi riscopre un ritorno inaspettato: la metafora, cioè una figura di unione e di tissage dopo tante azioni inverse. Figura madre di tutti i tropi, la metafora per definizione è una similitudo brevior, secondo Quintiliano, ovvero una figura che designa un oggetto attraverso un altro che ha col primo un rapporto di similitudine: non accentua la differenza che esiste fra due elementi ma, tramite un terzo (che scompare), accomuna due realtà assolutamente lontane. Eppure, così facendo, la differenza, aspetto tanto caro a Char come a Eraclito $^{62}$, è ancora una volta messa in vista.

Per funzionamento molto simile alla metonimia, la metafora obbedisce alla logica della sostituzione sempre restando sull'asse paradigmatico: la parola è declinata e dà luogo a diverse suggestioni, che, al di là del significato primo, conferiscono altrettante connotazioni allo stesso significato. Si scoprono così delle analogie impensate e lontane dalle associazioni logiche e spaziali quotidiane. Operante nel senso inverso alla metonimia, la metafora è una struttura che Char impara a conoscere nelle sue potenzialità quando milita giovanissimo nelle file del Surrealismo: qui la figura ha essenzialmente uno statuto distruttivo. Per l'artista qualcosa muterà dopo il distacco che lo porterà a non utilizzarla più gratuitamente, ovvero il comparante è adeguato al comparato: «"Les souris de l'enclume". Cette image m'aurait paru charmante autrefois. Elle suggère un essaim d'étincelles décimé en son éclair. (L'enclume est froide, le fer pas rouge, l'imagination dévastée) ${ }^{63}$. L'imagerie surrealista è respinta perché non abbastanza precisa e sostanzialmente vuota. Il commento di Char agisce sempre in base a un registro metaforico e corregge l'immagine iniziale sviluppandone un aspetto possibile, seppur lontano: allea animato e inanimato, astratto e concreto prevalentemente secondo due modi che Patrick Née ${ }^{64}$ ha ribattezzato "per interscambio fra metafore" e "per metafora filée".

Nel primo caso, due metafore semplici sono il punto di partenza per un'operazione non tanto di crispage su sé stesse (come spesso avviene nei testi chariani) ma di accorpamento in quanto entrano talmente in contatto fra loro da scambiarsi parzialmente il loro valore nell'economia di un sistema inglobante più complesso: «Au cours d'une lutte si noire et de l'immobilité si noire, la terreur aveuglant mon royaume, je m'élevai des lions ailés de la_moisson jusqu'au cri froid de l'anémone [...] $\gg^{65}$. Nel poème, si passa dai lions ailés de la moisson al cri froid de l'anémone tramite l'indicazione attiva del movimento di ascensione (je m'élevai des ... jusqu'au). Non vi è nulla nel grido freddo dell'anemone che denota un superare in elevazione; invece, la connotazione gli viene dalla contaminazione del sistema inglobante che associa all'emissione

(62) «Pour René Char, comme pour Héraclite [...] ce qui parle essentiellement dans les choses et dans les mots, c'est la Différence, secrète parce que toujours différant de parler et toujours différente de ce qui la signifie, mais telle aussi que tout fait signe et se fait signe à cause d'elle qui n'est dicible qu'indirectement, non pas silencieuse: à l'œuvre dans le détour de l'écriture» (M. BLANCHOT, Parole de fragment, L'entretien infini, Paris, Gallimard, 1980, p. 454 ).
(63) Note 52, Feuillets d'Hypnos, Fureur et mystère, OC, p. 187.

(64) P. NéE, Le poème en prose dans l'auvre de René Char, Actes du colloque international de l' Université de Tours sur René Char, tenu en juin 1983 sous la direction de D. LEUwERS, numéro spécial de la revue «Cahiers du Sud», Marseille, 1985, p. 138.

(65) Ne s'entend pas, Seuls demeurent, Fureur et mystère, OC, p. 142. 
vocale del cri (qualificato dall'altezza del tono), con l'aggiunta dell'idea di una zona stratosferica molto elevata (tanto da permettere lo svilupparsi "nel freddo" dal fiore del vento, ossia l'anemone), l'innalzamento dei leoni alati della moisson. Di converso, sempre per contaminazione, il freddo espresso nella seconda metafora provocherà l'avvicinamento del leone al grano nel campo semantico comune della natura.

Il secondo caso è una semplice sequela di metafore classiche che si susseguono au fil du texte assicurandogli una solidità semantica:

Tout le jour, assistant l'homme, le fer a appliqué son torse sur la boue enflammée de la forge. À la longue, leurs jarrets jumeaux ont fait éclater la mince nuit du métal à l'étroit sous la terre.

L'homme sans se hâter quitte le travail. Il plonge une dernière fois ses bras dans le flanc assombri de la rivière. Saura-t-il enfin saisir le bourdon glacé des algues? ${ }^{66}$

Nell'esempio, l'equivalenza esplicita del ferro e del muscolo umano conduce a un'evocazione del lavoro della fucina come lotta atletica («le fer a appliqué son torse; leurs jarrets jumeaux; [faire] éclater la mince nuit du métal e [plonger] une dernière fois ses bras dans le flanc assombri de la rivière»). La tendenza naturale delle metafore filées conduce direttamente alle allegorie, scandendo il testo in personificazioni e in drammatizzazioni di cui i poèmes sono disseminati (dalla Rencontre alla Nuit alla Sorgue, per citare le più famose).

La metafora, per sua natura, traduce una tessitura che rimanda alla scrittura della ruse mentre gli altri due tropi rimandano più alla scrittura della révolte. Si perpetua, così, l'opposizione già riscontrata a livello delle forme del contenuto: i versi, come prima i motivi, oscillano fra queste due posizioni senza mai risolversi in una decisiva.

Nelle intenzioni di Char, la metafora, rispetto a quella surrealista, non solo deve mostrare una realtà altra ma deve anche fare riflettere su questo nuovo possibile. Questo è lo stesso spirito che anima il funzionamento di una scrittura profondamente e consapevolmente vissuta. 